\title{
The auroral red line polarisation: modelling and measurements
}

Jean Lilensten ${ }^{1, *}$, Véronique Bommier ${ }^{2}$, Mathieu Barthélemy ${ }^{1}$, Hervé Lamy ${ }^{3}$, David Bernard ${ }^{1}$, Joran Idar Moen ${ }^{4,5}$, Magnar Gullikstad Johnsen ${ }^{6}$, Unni Pia Løvhaug ${ }^{7}$, and Frédéric Pitout ${ }^{8,9}$

1 UJF-Grenoble 1/CNRS-INSU, Institut de Planétologie et d'Astrophysique de Grenoble (IPAG), UMR 5274, 38041 Grenoble, France

*Corresponding author: jean.lilensten@obs.ujf-grenoble.fr

2 LESIA, CNRS, Observatoire de Paris, 92190 Meudon, France

3 Belgian Institute for Space Aeronomy, Ringlaan-3-Avenue Circulaire, 1180 Brussels, Belgium

4 Department of Physics, University of Oslo, P.O. Box 1048, 0316 Blindern, Oslo, Norway

5 University Centre in Svalbard, Longyearbyen, Norway

6 Tromsø Geophysical Observatory University of Tromsø, Norway

7 Department of Physics and Technology, University of Tromsø, Norway

8 Universit de Toulouse, UPS-OMP, IRAP, Toulouse, France

9 CNRS, IRAP, 9 Av. colonel Roche, BP 44346, 31028 Toulouse Cedex 4, France

Received 21 January 2014 / Accepted 21 June 2015

\begin{abstract}
In this work, we model the polarisation of the auroral red line using the electron impact theory developed by Bommier et al. (2011). This theory enables the computation of the distribution of the Degree of Linear Polarisation (DoLP) as a function of height if the flux of precipitated electrons is provided as input.

An electron transport code is used to infer the stationary electron flux at each altitude in the ionosphere as a function of energy and pitch angle. Using adequate cross-sections, the integral of this electron flux over energy and pitch angle provides an anisotropy parameter from which the theoretical local DoLP can be computed at each altitude. The modelled DoLP is then derived by integrating along the line-of-sight.

Depending on the integration length, the modelled DoLP ranges between $0.6 \%$ for a very long integration length and $1.8 \%$ for a very short integration length localised around an altitude of $210 \mathrm{~km}$. A parametric study is performed to check how the characteristics of the local DoLP (maximum value, altitude of the maximum, integrated height profile) vary. It is found that the polarisation is highly sensitive to the scattering function of the electrons, to the electron precipitation and to the geomagnetic activity. We compare these values to measured ones obtained during an observational campaign performed in February 2012 from Svalbard. The measured DoLP during the campaign was $1.9 \% \pm 0.1 \%$. The comparison between this value and the theoretical one is discussed. Discrepancies may be due to the poor constraint of the input parameters (thermosphere and ionosphere), to the fact that only electron precipitation is considered in this approach (and not proton precipitation for instance) and to the difficulty in constraining the exact width of the emission layer in the thermosphere.
\end{abstract}

Key words. Thermosphere - Auroral emissions - Polarisation

\section{Introduction}

The atomic oxygen red line is a triplet at $630.0,636.4$ and $639.2 \mathrm{~nm}$ and is one of the most intense lines in the auroral spectrum. It originates from the deactivation of the metastable $\mathrm{O}\left({ }^{1} \mathrm{D}\right)$ state. The polarisation of the thermospheric atomic oxygen red line at $630 \mathrm{~nm}$ was discovered by Lilensten et al. (2008) with a dedicated Steerable Photo Polarimeter (SPP) equipped with a narrow interference filter, and later confirmed by subsequent observations (Lilensten et al. 2013a). The Degree of Linear Polarisation (DoLP) observed has an average of $1-2 \%$, with maximum values smaller than $10 \%$.

In parallel, an important study has been carried out to compute the theoretical impact polarisation of the atomic oxygen red line (Bommier et al. 2011). The expected polarisation saturates at $17 \%$. For energies high above the red line excitation threshold of $1.97 \mathrm{eV}$, it is parallel to the incident beam of electrons, i.e., along the magnetic field line, for energies slightly above (but close to) the threshold it is perpendicular to the magnetic field (see Fig. 2 of Bommier et al. 2011). Electrons are assumed to precipitate along the magnetic field line. Theory and observations cannot be easily compared because first we need to take into account an anisotropic stationary flux of electrons precipitating in the ionosphere (with some pitch angles to the magnetic field) and second there are a number of competing mechanisms leading to the formation of the red line that do not produce polarisation and therefore act as depolarising mechanisms. The main goal of this article is to solve these two issues and to model the polarisation of the red line by combining the theoretical study described above with a kinetic electron transport code. In Section 2, we review the most important aspects of the theory of the impact polarisation of the red line. The theory is then generalised to take into account an anisotropic flux of precipitating electrons (in terms of pitch angles). Finally, the kinetic electron transport code, transsolo, is briefly presented with discussions regarding inputs and outputs. In Section 3, these outputs are used in the generalised theory to obtain a profile of the red line polarisation along the magnetic field. 
A parametric study is then presented to evaluate the relative influences of each parameter. In Section 5, we attempt to compare the predictions of this model to observations of the red line polarisation obtained with SPP during a campaign organised in February 2012 in Longyearbyen in the Svalbard archipelago and presented in Section 4.

Since there is sometimes some ambiguity in the vocabulary used in the literature, we remind that in the following, the ionosphere represents the ionised part of the upper atmosphere (ions and electrons), the thermosphere represents its neutral component and the upper atmosphere is the mixture of the ionosphere and the thermosphere.

\section{Theory of linear polarisation}

\subsection{Theory of the polarisation}

The Degree of Linear Polarisation (DoLP) is the ratio of the Stokes parameters $Q$ and $I$. Charvin (1965) and Sahal-Bréchot (1974) have shown (in the case of the forbidden green line of the solar corona) that it can be expressed as a function of a unique parameter $\eta$, as:

$$
\operatorname{DoLP}=\frac{Q}{I}=\frac{3 \eta \sin ^{2} \Theta}{1-\eta\left(1-3 \cos ^{2} \Theta\right)},
$$

for a dipolar magnetic emission like the auroral red line, for a reference $\mathrm{O} x$ axis lying in the scattering plane defined by the impact incident direction and the line-of-sight $\mathrm{O} z$, referred to with the scattering angle $\Theta$. $Q$ is positive when the linear polarisation direction lies in the scattering plane, along the Ox axis. Equation (1) is the local DoLP, which would be derived for a single scattering point. The line-of-sight integration will be considered later in the paper. As the observations are made at right-angle scattering (Sect. 4.4), in the following we present local DoLP values computed with a right angle between the line-of-sight and the local magnetic field line (i.e. $\Theta=90^{\circ}$ ). In the case of collisional excitation such as those at work in this study, $\eta$ is proportional to the ratio of the upper level atomic density matrix elements.

$$
\eta=C_{\eta} \frac{{ }^{A} \rho_{0}^{2}}{{ }^{A} \rho_{0}^{0}},
$$

where $C_{\eta}$ depends on the level quantum numbers as

$$
C_{\eta}=\frac{1}{2 \sqrt{2}} w_{J^{\prime} J}^{(2)}
$$

where

$$
w_{J^{\prime} J}^{(2)}=(-1)^{1+J+J^{\prime}} \sqrt{3\left(2 J^{\prime}+1\right)}\left\{\begin{array}{lll}
1 & J^{\prime} & J \\
J^{\prime} & J & 2
\end{array}\right\} .
$$

This coefficient, which is related to the line polarisability, has been introduced and tabulated by Landi Degl'Innocenti (1984), and includes a 6-j symbol (see, for instance, Brink \& Satchler 1994). In the case of the auroral oxygen red line considered here, $J=J^{\prime}=2$ and $C_{\eta}$ is equal to $-\frac{1}{4} \sqrt{\frac{7}{10}}=-0.209165$. For the atomic density matrix elements, $\sqrt{2 J^{\prime}+1}\left({ }^{A} \rho_{0}^{2}\right)$ is the normalised upper level alignment, whereas $\sqrt{2 J^{\prime}+1}\left({ }^{A} \rho_{0}^{0}\right)$ is the normalised upper level population. The density matrix elements are developed in irreducible tensorial components $\rho_{q}^{k}$ (see, for instance,

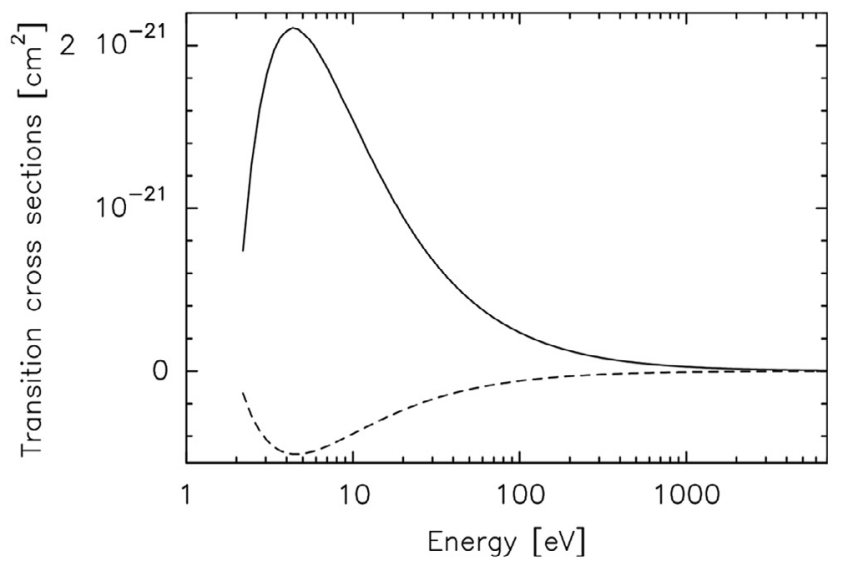

Fig. 1. Alignment creation cross-section $\sigma(0 \rightarrow 2)$ (dashed line) and excitation cross-section $\sigma(0 \rightarrow 0)$ (full line).

Landi Degl'Innocenti \& Landolfi 2004, for definitions). The upper index $A$ stands for "initial reference frame", because a frame rotation will be introduced below. The initial reference frame is the one defined by the impact geometry, with $\mathrm{O} z$ quantisation axis along the incident electron trajectory.

The parameter $\eta$ can be expressed in terms of the alignment creation cross-section $\sigma(0 \rightarrow 2)$ and excitation crosssection $\sigma(0 \rightarrow 0)$, for a given impacting electron and an isolated oxygen atom:

$$
\eta=C_{\eta} \frac{\sigma(0 \rightarrow 2)}{\sigma(0 \rightarrow 0)}
$$

where the impact transition probability is given by

$$
p\left(J \rightarrow J^{\prime}\right)=\frac{\sqrt{2 J^{\prime}+1}}{\sqrt{2 J+1}} \sigma(0 \rightarrow 0),
$$

due to normalisation. These cross-sections are computed following the method developed by Bommier et al. (2011) for this forbidden line, where the interaction potential with the incoming electron is quadrupolar electric. The alignment creation cross-section $\sigma(0 \rightarrow 2)$ and the excitation cross-section $\sigma(0 \rightarrow 0)$ are plotted in Figure 1. The algebraic value of $\sigma(0 \rightarrow 2)$ may be negative, because the alignment itself may be negative, being a difference between Zeeman sublevel populations. This is the case for the forbidden oxygen red line.

In the case of the thermospheric oxygen for the red line, other processes compete with the electron impact to populate the upper level of the line but do not produce polarisation. They are listed in the next section describing the kinetic electron transport code. Since the $\eta$ coefficient is proportional to the ratio of upper level alignment to upper level population, it has to be weighted by the relative importance of the electron impact with respect to all these processes. We denote as $R_{\text {coll }}$

$$
R_{\text {coll }}(z)=\frac{P_{\text {coll }}(z)}{P_{\text {tot }}(z)},
$$

the ratio between $P_{\text {coll }}$, which is the red line emission intensity produced through collisions of atomic oxygen with electrons, and $P_{\text {tot }}$, which is the red line emission intensity produced by all processes considered (listed in Sect. 2.2):

$$
R_{\mathrm{coll}}(z)=\frac{P_{1}(z)+P_{4}(z)}{\sum_{k=1}^{7} P_{k}(z)} .
$$


In other words, each density matrix component is determined by an equilibrium between gains and losses, which can be written as:

$$
L_{\text {tot }} \sqrt{2 J^{\prime}+1^{A}} \rho_{0}^{0}=P_{\text {tot }},
$$

for the population, and

$$
L_{\text {tot }} \sqrt{2 J^{\prime}+1^{A}} \rho_{0}^{2}=P_{\text {coll }} \frac{\sigma(0 \rightarrow 2)}{\sigma(0 \rightarrow 0)},
$$

for the alignment, where $L_{\text {tot }}$ is the total probability of leaving the level. All these processes destroy the level alignment and population as well. Due to the weak densities at the altitude of the red line peaks, there is no "depolarising collision" process, destroying the alignment but not the population, by collisional transitions between the Zeeman sublevels. As a consequence, the ratio of density matrix elements is locally, at equilibrium:

$$
\frac{{ }^{A} \rho_{0}^{2}}{{ }^{A} \rho_{0}^{0}}=R_{\text {coll }} \frac{\sigma(0 \rightarrow 2)}{\sigma(0 \rightarrow 2)} .
$$

All the quantities $P_{\text {coll }}, P_{\text {tot }}$ and $R_{\text {coll }}$ depend on the altitude $z$.

Finally, the incoming electrons are only partially directive. They are not all aligned with the magnetic field line and have some pitch angle distribution. We assume that they obey an axial symmetry about the magnetic field. If we denote $\left(x^{\prime}, y^{\prime}\right.$, $\left.z^{\prime}\right)$ as the initial or single-electron impact reference frame with $\mathrm{O} z^{\prime}$ axis along one electron velocity, and $(x, y, z)$ the final reference frame with $\mathrm{O} z$ axis along the magnetic field, and if $(\theta, \varphi)$ denotes the polar and azimuth angles, respectively, of the elementary $\mathrm{O} z^{\prime}$ axis in the $(x, y, z)$ reference frame, the density matrix elements in the "new" frame $(x, y, z),{ }^{N} \rho_{q}^{k}$ are related to those in the elementary frame ${ }^{A} \rho_{q}^{k}$ by:

$$
{ }^{N} \rho_{q}^{k}=\sum_{q^{\prime}} \mathrm{e}^{-\mathrm{i} \varphi q} d_{q^{\prime} q}(-\theta) \rho_{q^{\prime}}^{k}
$$

where $d q^{\prime} q(-\theta)$ is a Euler rotation matrix element (see, for instance, Brink \& Satchler 1994). In the $\varphi$ integration due to the axial symmetry, the $q \neq 0$ elements vanish due to:

$$
\int_{0}^{2 \pi} \mathrm{e}^{-\mathrm{i} \varphi q} \mathrm{~d} \varphi=\delta_{q 0}
$$

where $\delta_{q 0}$ is the Kronecker symbol, so that finally only remains $d_{00}(-\theta)$, which is $\frac{1}{2}\left(3 \cos ^{2} \theta-1\right)$. The parameter $\eta$ can now be generalised as:

$$
\eta(z)=C_{\eta} A(z) R_{\text {coll }}(z)
$$

where $A(z)$ is an anisotropy factor defined by:

$$
A(z)=\frac{\iint \mathrm{d} E \mathrm{~d} \theta \sin \theta \Phi(z, E, \theta) \frac{1}{2}\left(3 \cos ^{2} \theta-1\right) \sigma(0 \rightarrow 2)}{\iint \mathrm{d} E \mathrm{~d} \theta \sin \theta \Phi(z, E, \theta) \sigma(0 \rightarrow 0)},
$$

where $\Phi(z, E, \theta)$ is the incoming electron flux at altitude $z$, energy $E$ and at the pitch angle $\theta$ with respect to the magnetic field. $A(z)$ may be interpreted as an integrated anisotropy (integrated over all energies and pitch angles). The contribution of a given energy $E$, disregarding the cross-sections, is simply the anisotropy degree $a(z, E)$ of the incident electron beam:

$$
a(z, E)=\frac{\sin \theta \Phi(z, E, \theta) \frac{1}{2}\left(3 \cos ^{2} \theta-1\right)}{\sin \theta \Phi(z, E, \theta)} .
$$

The anisotropy $a(z, E)$ varies from 0 (full isotropy) to 1 . Finally, for comparison with the observations, the DoLP has to be integrated along the line-of-sight. This has to be done for $I$ and $Q$, respectively, and, in an optically thin medium like the aurora, the integrated DoLP results in:

$$
\langle\operatorname{DoLP}\rangle=\frac{\int_{z_{\min }}^{z_{\max }} Q(z) \mathrm{d} z}{\int_{z_{\min }}^{z_{\max }} I(z) \mathrm{d} z},
$$

where $Q$ and $I$ are the usual Stokes parameters and where the integration limits $z_{\min }$ and $z_{\max }$ correspond to the aurora boundaries, whose determination in our present observation will be discussed in Section 5.2. Although $\langle$ DoLP $\rangle$ is a ratio of integrals, we call it the line-of-sight integrated DoLP. In contrast, in the following we will denote as "local DoLP" the one coming from Eq. (1).

\subsection{Kinetic electron transport code}

The computation of the anisotropy factor requires to know $\Phi(z, E, \theta)$. This stationary electron flux can be computed using a kinetic electron transport code called transsolo. The first version of this code is described in Lummerzheim \& Lilensten (1994). Since then, it has been constantly improved and used in different applications. A more recent description is given in Lilensten \& Cander (2003) and the latest developments are mentioned in Lilensten et al. (2013b). Details will not be given here but we describe hereafter the concept of the code.

A stationary kinetic Boltzmann transport equation is solved along the magnetic field line to obtain the electron flux $\Phi(z, E$, $\theta$ ) (in $\mathrm{cm}^{-2} \mathrm{~s}^{-1} \mathrm{eV}^{-1} \mathrm{sr}^{-1}$ ) at each altitude $z$, energy $E$ and pitch angle $\theta$. At nighttime, the inputs of this model are the neutral atmosphere, the electron temperature and density profiles, the electron impact cross-sections and the flux of precipitating electrons at the top of the ionosphere. The full bibliography for the cross-sections is given in Lilensten \& Cander (2003). The neutral atmosphere is provided by the empirical model NRLMSISE-00, Picone et al. (2002). The electron temperature and density profiles can be obtained e.g. using incoherent scatter radar data. Following Rees (1989), we impose a flux of precipitating electrons made of two components: a Maxwellian at a suprathermal energy (typically a few $\mathrm{keV}$ ) and a power law below $10 \mathrm{eV}$. This flux enters the upper atmosphere with a given pitch angle distribution. The bulk of the particles is directed along the local magnetic field line. The flux of particles decreases gradually from this parallel direction and becomes zero in the direction perpendicular to the magnetic field. Only the characteristic energy (peak) of the Maxwellian and the total integrated energy flux are free parameters.

The code provides the stationary electron flux $\Phi(z, E, \theta)$ needed to compute the anisotropy, but it also provides the emission line intensities which are necessary to compute the polarisation itself. Indeed, the denominator in Eq. (7) denotes the red line intensity due to production of excited oxygen through different mechanisms which are described in Witasse et al. (1998) and references therein:

- p1: Suprathermal electron impact on O. p1 directly depends on $\Phi(z, E, \theta)$ through:

$$
p 1(z)=[\mathrm{O}](z) \int \sigma_{1 D}(E) \Phi(z, E, \theta) \mathrm{d} E \mathrm{~d} \theta,
$$


where $z$ is the altitude, $[\mathrm{O}](z)$ is the atomic oxygen density at altitude $z, E$ is the energy, and $\sigma_{1 \mathrm{D}}(E)$ is the excitation crosssection.

- p2: Dissociative recombination of $\mathrm{O}_{2}{ }^{+}$

- p3: $\mathrm{O}_{2}$ photodissociation

- p4: Thermal electron impact on O. Thermal electrons have a Maxwellian energy distribution with typical characteristic energy of less than $1 \mathrm{eV}$.

- p5: Cascading from $\mathrm{O}\left({ }^{1} \mathrm{~S}\right)$ state

- p6: $\mathrm{N}\left({ }^{2} \mathrm{D}\right)+\mathrm{O}_{2}$

- p7: $\mathrm{N}^{+}+\mathrm{O}_{2}$

The red line emission is computed through the balance between all production and loss mechanisms of the $\mathrm{O}\left({ }^{1} \mathrm{D}\right)$ state. The loss mechanisms are:

- 11: Radiative transitions (red line emission at 630, 636.3, and $639.1 \mathrm{~nm}$, Witasse et al. 1998)

- 12: Quenching by $\mathrm{O}_{2}$

- 13: Quenching by $\mathrm{O}$

- 14: Quenching by $\mathrm{N}_{2}$

- 15: Quenching by thermal electrons

The impact with suprathermal electrons (p1) and the impact with thermal electrons (p4), which have usually energies smaller than $1 \mathrm{eV}$, constitute the most important sources of the auroral nightglow. Only the first process is anisotropic because the presence of the magnetic field imposes a collimated pitch angle distribution and therefore it contributes strongly to create the anisotropy in Eq. (15). The thermal electrons are isotropic and play no role in Eq. (15). Therefore, among the two sources, only impact with energetic electrons is expected to give rise to polarisation. However, the thermal electrons can in principle also modify the polarisation through $R_{\text {coll }}$ term in Eq. (14). The numerator of $R_{\text {coll }}$ (Eq. (7)) represents the production of the red line through collisions. It is equal to the sum of $\mathrm{p} 1$ and $\mathrm{p} 4$.

\section{Modelling of the polarisation}

In this section, we combine the outputs of the transsolo model with the theory of the polarisation in order to model the polarisation profile of the red line. To start, the input parameters used in the transport code will be chosen as close as possible to those observed during coordinated observations of SPP and the EISCAT Svalbard radar, both located in Longyearbyen, in the Svalbard Archipelago ( $78^{\circ}$ geographic north latitude), during the night from February 15-16, 2012, from $0: 33^{\prime} 43^{\prime \prime}$ LT to $0: 39^{\prime} 19^{\prime \prime}$ LT. In this first reference test case, the flux of precipitating electrons will consist in a suprathermal Maxwellian distribution centred around $1 \mathrm{keV}$ with a total integrated energy flux of $0.02 \mathrm{erg} \mathrm{cm}^{-2} \mathrm{~s}^{-1}$ or $1.25 \times 10^{10} \mathrm{eV} \mathrm{cm}^{-2} \mathrm{~s}^{-1}$ (see Fig. 2). The choice of these parameters will be justified in Section 4. The NRLMSISE-00 model is used to specify neutral atmosphere characteristics for that specific day and time, the geographical coordinates of Longyearbyen and values of $F_{10.7}=105$ and $A p=21$ (Fig. 3). Finally, the electron temperature and density profiles to start will be measured by the EISCAT Svalbard radar (Fig. 4). Details of these observations are given in Section 4.

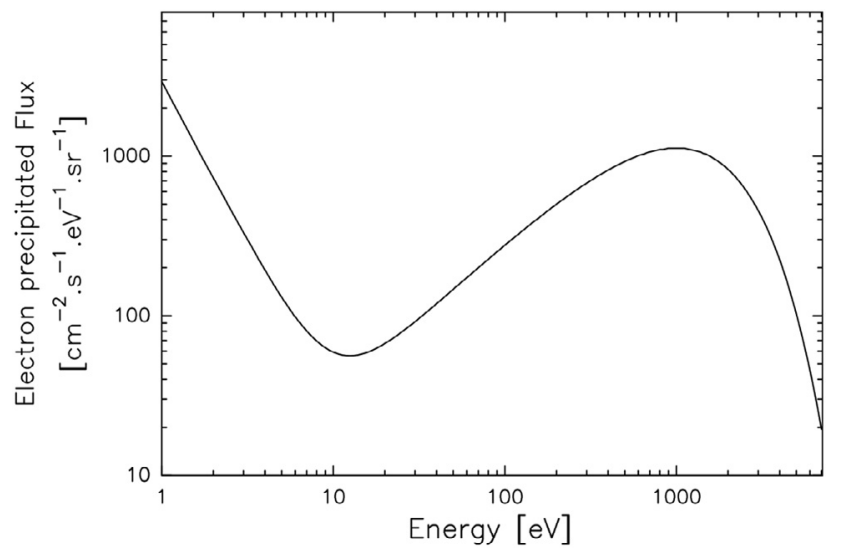

Fig. 2. Flux of precipitating electrons used for the reference test case.

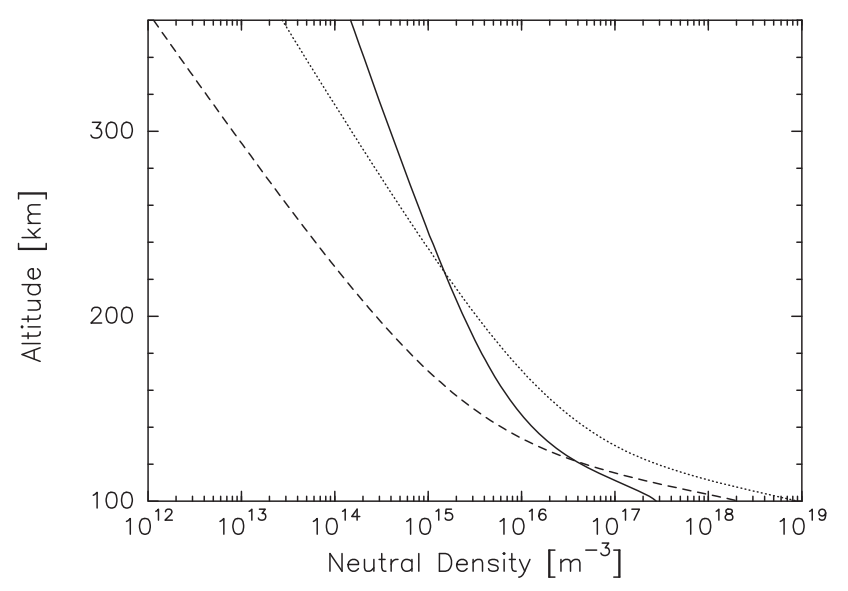

Fig. 3. Neutral atmosphere provided by NRLMSISE-00 for the reference test case, $\mathrm{O}$ (straight line), $\mathrm{O}_{2}$ (dashed line), $\mathrm{N}_{2}$ (dotted line).

For this reference test case and in the following, we use 101 altitudes between 90 and $500 \mathrm{~km}, 200$ energies from $0.1 \mathrm{eV}$ to $7000 \mathrm{eV}$ and 32 pitch angles.

\subsection{Red and green line intensities}

The computed intensity profiles of the red and green lines are shown in Figure 5. The red line emission peaks at about $220 \mathrm{~km}$ and the green line emission at $110 \mathrm{~km}$, which are typical features observed at these high latitudes (Solomon \& Abreu 1989; Culot et al. 2005). In Figure 6, the production rates of the red line are shown for both thermal and suprathermal electrons. At all altitudes, the contribution of thermal electrons is much smaller than that due to suprathermal electrons, by a factor larger than 100 . Only at very low altitude $(\sim 100 \mathrm{~km})$ the contribution due to thermal electrons dominates. The $\mathrm{O}^{1} \mathrm{D}$ production rate peaks at low altitude, around $110 \mathrm{~km}$, whilst the red line emission peaks at much higher altitude, typically around $220 \mathrm{~km}$. This difference is explained by the different losses through collisions with the neutral gas and the ambient electrons (Witasse et al. 1998, 1999; Culot et al. 2005). 


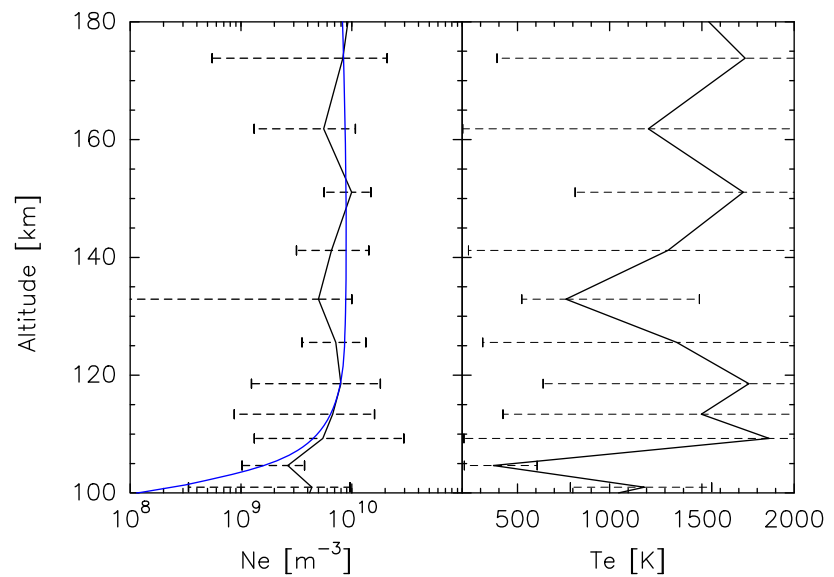

Fig. 4. Left panel: measured electron density profile (black line) and the fit used in the test case of the theoretical study. Right panel: measured electron temperature profile, used in the test case of the theoretical study.

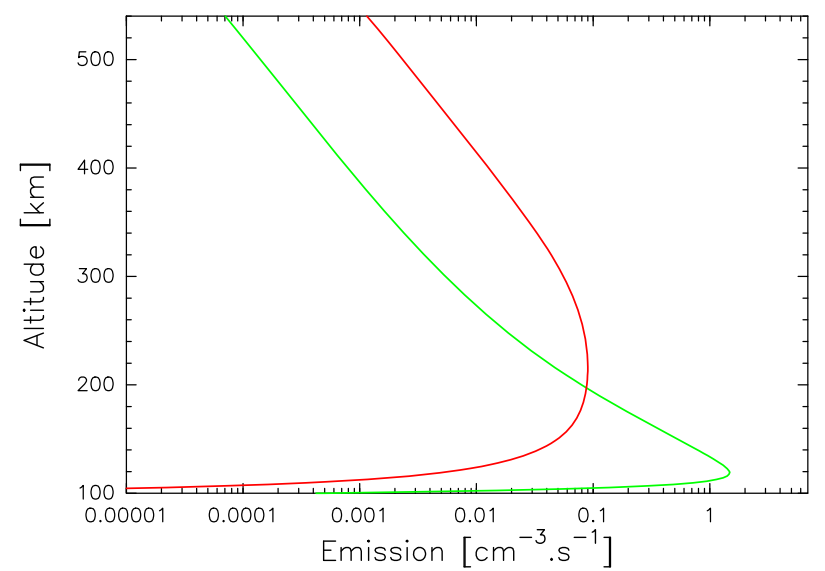

Fig. 5. The red and green line intensity profiles computed by the electron transport code for the reference test case.

\subsection{Polarisation profile of the red line}

In Figure 7, we show $A(z)$ (Eq. (15)), the ratio $R_{\text {coll }}=\frac{P_{\text {coll }}(z)}{P_{\text {tot }}(z)}$ (Eq. (14)) and the local DoLP (Eq. (1)). The anisotropy maximises at about $210 \mathrm{~km}$ height with a value of about 0.02 , i.e. approximately the same altitude where the red line emission maximises. The shape of $A(z)$ strongly depends on the shape of the stationary electron flux integrated on the pitch angle and energy. At high altitude, the part of the flux at energies below a few $\mathrm{eV}$ is small and therefore the energy integral at these low energies, where the ${ }^{1} \mathrm{D}$ state is excited, is small as well. At low altitude, most of the energy of the input flux has been dissipated through ionisation, excitation, dissociation or heating and therefore $A(z)$ tends to 0 . The ratio $R_{\text {coll }}$ includes in principle contributions from both thermal and suprathermal electrons. Since the contribution of the thermal electrons is negligible, it only depends on the suprathermal electrons. If only thermal electrons are taken into account in the modelling, the local DoLP maximises at $186 \mathrm{~km}$ altitude, but with a totally negligible amplitude of $\sim 0.001 \%$. The modelled local DoLP is maximum at $213 \mathrm{~km}$ with a value of $1.79 \%$. Its value integrated between 90 and $500 \mathrm{~km}$ along the line-of-sight is equal to $0.62 \%$. With all the uncertainties in the input

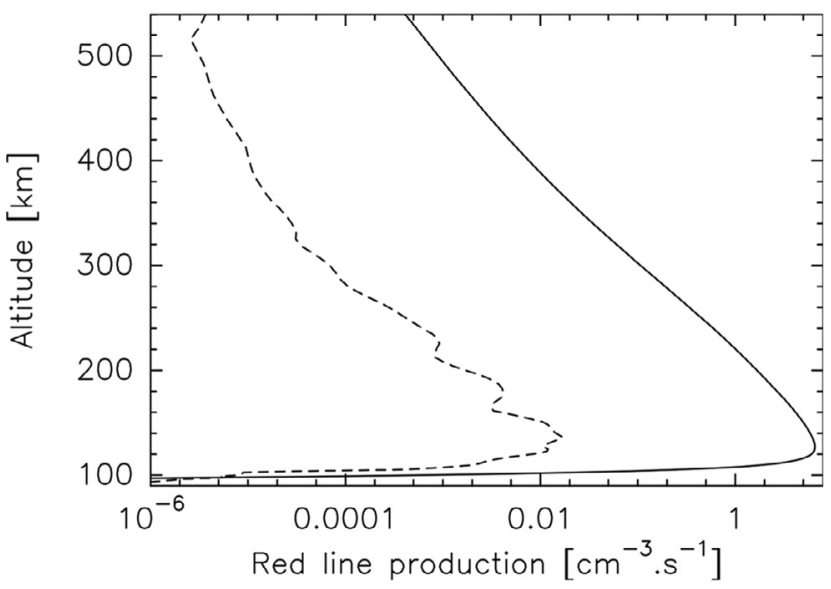

Fig. 6. Contribution to the red line production rate by the suprathermal electrons (full line) and the thermal electrons (dashed line). The latter is not as smooth as the former because of the discontinuities in the measured electron temperatures and densities.

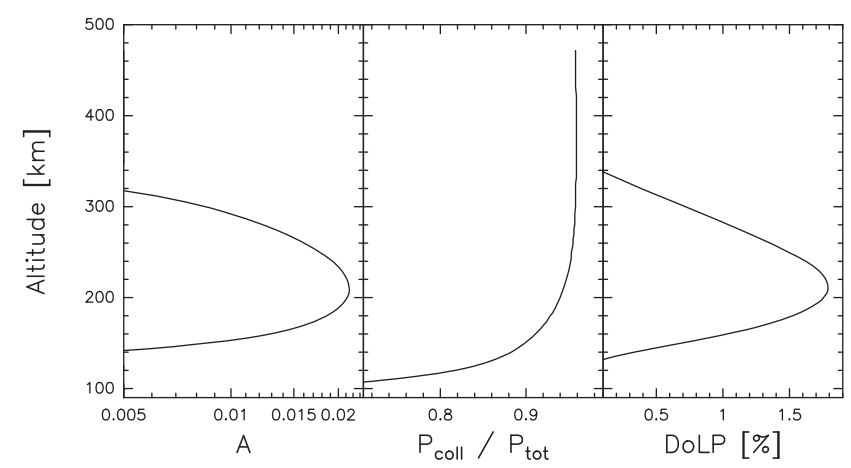

Fig. 7. Results of the reference test case. Left panel: the anisotropy parameter $A(z)$ (unitless). Middle panel: the ratio $R_{\text {coll }}$ (unitless). Right panel: local Degree of Linear Polarisation (DoLP) in \%.

parameters, we will use, respectively, $1.8 \%$ and $0.6 \%$ in the comparisons below.

\subsection{Sensitivity to input parameters}

In the following paragraphs, we depart from the test case and study the influence of several parameters of the transsolo model on the results shown in Figure 7. We summarise how the DoLP characteristics (maximum amplitude, altitude of the maximum and height-integrated value) vary in Table 1 and compare them to the test case of the previous section.

\subsubsection{Effect of the scattering phase function}

When an electron elastically collides with a target (i.e. the thermospheric gas made of $\mathrm{N}_{2}, \mathrm{O}_{2}$ and $\mathrm{O}$ ), it is scattered along an angle given by a scattering phase function. This function is rather poorly known and has very seldom been discussed in any ionospheric paper (Lummerzheim et al. 1989). In the Boltzmann kinetic equation, this function appears as part of the differential cross-section, which is the formalism describing how a suprathermal electron loses energy and changes its pitch angle during a collision. We refer the reader 
Table 1. Summary of the influence of the parameters on the DoLP. Column 1 explains the changes made to the test case. The results for the test case are given in the first line. Columns 2 and 3 are the altitude at which the local DoLP maximises and the percentage change compared with the test case, respectively. Columns 4 and 5 are the maximum local DoLP and percentage change, respectively. Columns 6 and 7 are the lineof-sight integrated DoLP and the percentage change, respectively. The relevant figure for each result is given in column 9.

\begin{tabular}{|c|c|c|c|c|c|c|c|}
\hline Change vs. test & Alt & $\%$ & $\max$ & $\%$ & Int & $\%$ & Fig. \\
\hline Test case & 213 & & 1.8 & & 0.6 & & 7 \\
\hline Only thermal $\mathrm{e}^{-}$ & 186 & -12.7 & 0. & - & 0.001 & - & - \\
\hline Only supratherm. & 213 & 0 & 1.79 & 0 & 0.62 & 0 & - \\
\hline Isotropic ph. fct. & 325 & 52.6 & 0.58 & -67.6 & 0.46 & -25.5 & 8 \\
\hline Forward ph. fct. & 208 & -2.3 & 2.03 & 13.4 & 0.63 & 1.6 & 8 \\
\hline$[\mathrm{O}] / 2$ & 193 & -9.4 & 1.99 & 11.2 & 0.76 & 22.6 & 9 \\
\hline$[\mathrm{O}] * 2$ & 244 & 14.6 & 1.77 & -1.1 & 0.51 & -17.7 & 9 \\
\hline$\left[\mathrm{N}_{2}\right] / 2$ & 208 & -2.3 & 1.81 & 1.1 & 0.58 & -6.4 & 9 \\
\hline$\left[\mathrm{N}_{2}\right]^{* 2}$ & 217 & 1.9 & 1.83 & 2.2 & 0.68 & 9.7 & 9 \\
\hline$\left[\mathrm{O}_{2}\right] / 2$ & 213 & 0 & 1.76 & -1.7 & 0.61 & -1.6 & 9 \\
\hline$\left[\mathrm{O}_{2}\right]^{* 2}$ & 213 & 0 & 1.83 & 2.2 & 0.64 & 3.2 & 9 \\
\hline Storm & 243 & 14.1 & 1.76 & -1.7 & 0.77 & 24.2 & 9 \\
\hline 1 erg prec. & 213 & 0 & 1.79 & 0 & 0.62 & 0 & - \\
\hline$\left[\mathrm{N}_{\mathrm{e}}\right] / 2$ & 213 & 0 & 1.83 & 2.2 & 0.64 & 3.2 & 10 \\
\hline$\left[\mathrm{N}_{\mathrm{e}}\right]^{*} 2$ & 209 & -1.9 & 1.73 & -3.4 & 0.60 & -3.2 & 10 \\
\hline $\mathrm{T}_{\mathrm{e}} / 2$ & 213 & 0 & 1.79 & 0 & 0.62 & 0 & 10 \\
\hline $\mathrm{T}_{\mathrm{e}} * 2$ & 209 & -1.9 & 1.89 & 5.6 & 0.61 & -1.6 & 10 \\
\hline Isotropic prec. & 221 & 3.8 & 2.07 & 15.6 & 0.75 & 21.0 & 11 \\
\hline Polar rain & 225 & 5.6 & 2.34 & 30.7 & 0.69 & 11.3 & 11 \\
\hline
\end{tabular}

to Lummerzheim \& Lilensten (1994) and Lilensten \& Cander (2003) for additional details. Here we focus only on the scattering phase function.

Porter et al. (1987) made a careful analysis of the scattering function for electron energies above $300 \mathrm{eV}$ in pure $\mathrm{N}_{2}$ gas. Since then, the Porter function has been used also for other atmospheric species and lower energies without reconsidering its validity. In this study, we will use the Porter scattering phase function for $\mathrm{N}_{2}$ and $\mathrm{O}_{2}$. However, the properties of the oxygen atoms (electric/magnetic properties, interactions, mass, symmetry properties) for electron collisions are too different from those for $\mathrm{N}_{2}$ (Moore 1993). Also, the energies in Porter et al. (1987) do not coincide with those at work for the red line emission.

During the last decades, the Henyey-Greenstein function has been used. This function was introduced in 1941 (Wiscombe \& Gums 1998) and is given by:

$$
p(\mu)=\frac{1}{2} \frac{1-g^{2}}{\left(1+g^{2}-2 g \mu\right)^{\frac{3}{2}}},
$$

where $\mu$ represents the cosine of the scattering angle. By varying the parameter $g(-1 \leq g \leq 1)$, this function ranges from backscattering through isotropic scattering $(g=0)$ to forward scattering. For $g>0$, forward scattering is dominant whilst for $g<0$ backscattering predominates. The function is normalised such that the integral over $4 \pi$ steradians is 1 and therefore it naturally conserves the total number of particles. It is interesting to note that this function was first used to describe photon scattering, and that using it for particles has no theoretical basis. Forward scattering corresponds to $\mu=1$. There are several ways of coding the scattering function, but for the ionospheric applications, it is natural to make $g$ vary as a function of the electron energy. At high energy (typically above $1 \mathrm{keV}$ ), the scattering is mostly forward (Opal et al. 1971). At thermal energies, the scattering is fully isotropic. How $g$ varies in between is unknown.

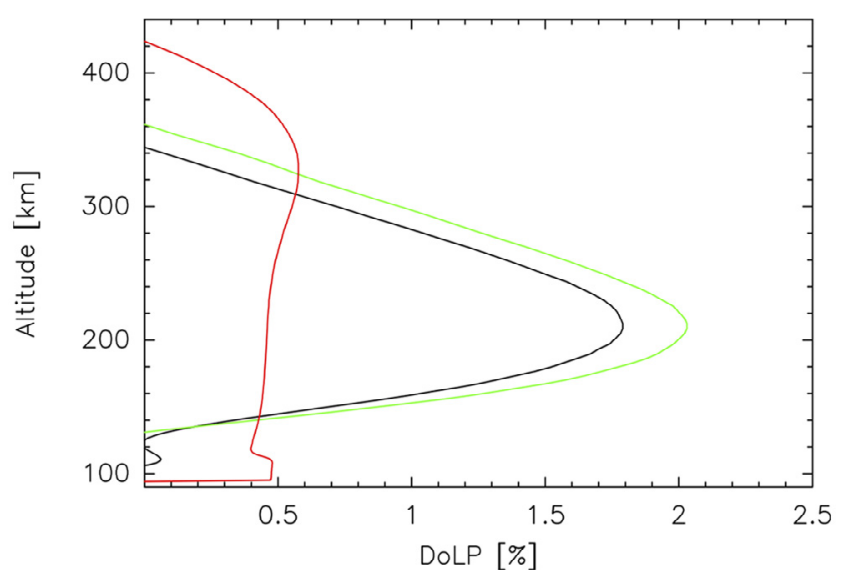

Fig. 8. Influence of the scattering function on the local DoLP. The black continuous curve is the reference test case as in Figure 7, right panel. The green curve corresponds to a fully forward scattering case and the red to the isotropic case.

In the present calculation, we use the Porter phase function for $\mathrm{N}_{2}$ and $\mathrm{O}_{2}$, and the Henyey-Greenstein described above for the atomic oxygen. We vary the energy at which the forward and isotropic contributions are equal. In the previous section for our test case, this energy has been set arbitrarily to $1.97 \mathrm{eV}$, i.e. the threshold energy for the red line production through electron collisions. This is how a value of $1.8 \%$ at $213 \mathrm{~km}$ was obtained for the local DoLP. This value is contained between two extreme cases: a fully isotropic scattering function and a strictly forward scattering one (for the suprathermal electrons). The results are shown in Figure 8. The isotropic scattering case gives a DoLP which maximises at $325 \mathrm{~km}$ with a peak value of $0.58 \%$. The altitude distribution is quite flat though, with an enhancement below $100 \mathrm{~km}$ due to the thermal electron contribution. In that case, the anisotropy 


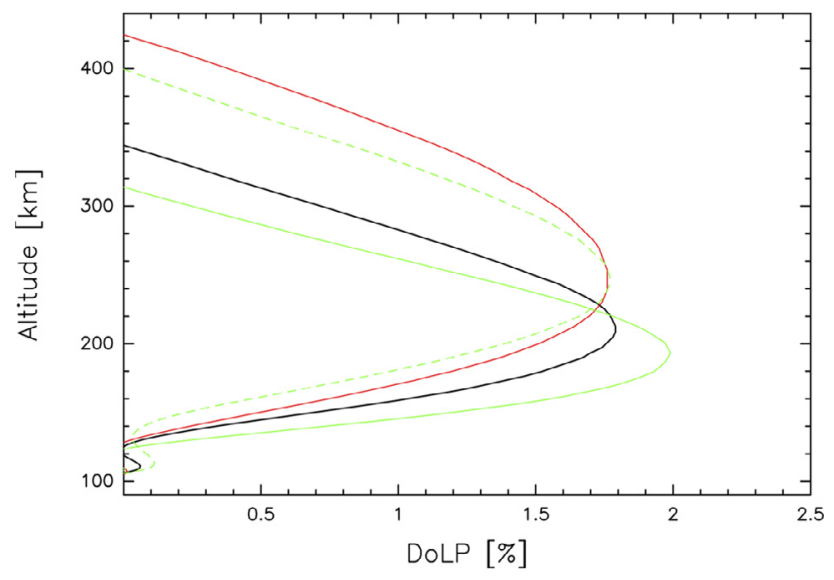

Fig. 9. Influence of the neutral atmosphere on the local DoLP. The black continuous curve is the reference test case as in Figure 7, right panel. The green lines are the effect of dividing (continuous line) or multiplying (dashed line) the atomic oxygen by 2 . The red line corresponds to geomagnetic storm conditions.

giving rise to the polarisation is only due to the fact that the precipitating electrons enter the atmosphere downward. The strictly forward scattering case gives a maximum value of the DoLP of $2.03 \%$ at $208 \mathrm{~km}$ with a well-peaked altitude distribution.

From the above study, we learn that if the neutral atmosphere is well known, the measurement of the DoLP of the red line may provide information on the electron scattering phase function for electron collisions with atomic oxygen, a function that is largely unknown up to now. The height of the peak of the DoLP may be one parameter to use in determining this scattering function.

\subsubsection{Effect of the neutral atmosphere}

The neutral atmosphere is one of the external parameters in the modelling. The results depend directly on the oxygen density, but also on the other neutral species which modify the stationary electron flux through the collisions of the atomic oxygen with the other species.

Many efforts have been devoted in the last years to improve the thermospheric models. Bruinsma et al. (2012) show a comparison between DTM2000 and MSIS. The discrepancy between them may be large, especially at high altitude $(>400 \mathrm{~km})$ where the atomic oxygen is preponderant, and where the ratio between models can reach a factor of 2 . Comparisons with measurements (in that case, the CHAMP satellite) also show large differences. Therefore, we first divided or multiplied independently the densities of $\mathrm{N}_{2}, \mathrm{O}_{2}$ and $\mathrm{O}$ by a factor of 2 . The results when dividing or multiplying the $\mathrm{O}$ density by 2 are shown in Figure 9. As expected, the effect is rather strong and visible at all altitudes. The altitude of the peak local DoLP experiences a large variation, from 193 (division by a factor of 2) to $244 \mathrm{~km}$ (multiplication by a factor of 2). The amplitude of the maximum local DoLP varies from $1.99 \%$ (division by a factor of 2) to $1.77 \%$ (multiplication by a factor of 2 ). The results when dividing or multiplying the densities of $\mathrm{N}_{2}$ and $\mathrm{O}_{2}$ are not shown in Figure 9 because the effects on both the maximum value of the DoLP and the altitude of the maximum are not significant (see Table 1).

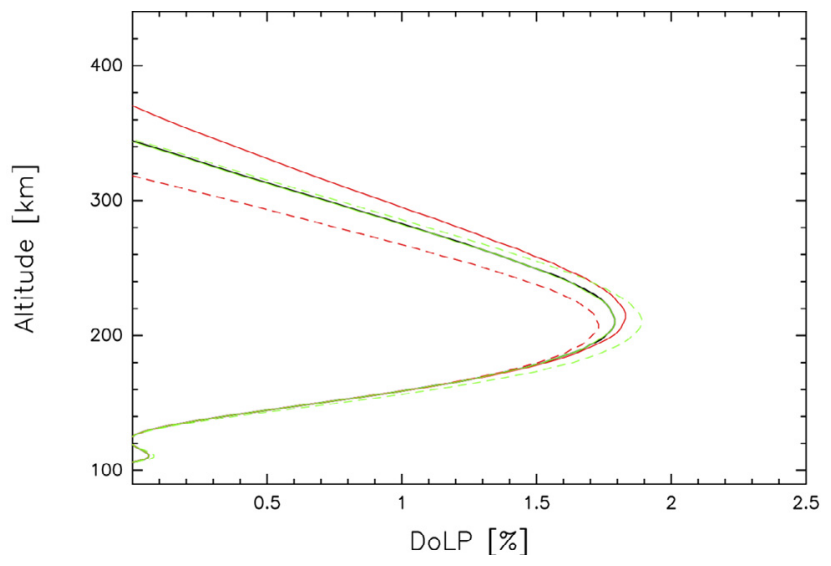

Fig. 10. Influence of the electron temperature and density on the local DoLP. The black continuous curve is the same as in Figure 7, right panel. It is hardly visible because it is almost covered by the green continuous lines which is the estimate of the DoLP when the electron temperature is divided by a factor of 2 . The green dashed line corresponds to a multiplication of 2 of the electron temperature. The red lines show the effect of dividing (continuous line) the electron density by 2 or multiplying (dashed line) it by 2 .

Next, we have simulated the effect of a magnetic storm by using $\mathrm{F}_{10.7}=300$ instead of 105 , and $\mathrm{Ap}=150$ instead of 21 in the MSISE-00. Results are shown in Figure 9 and the effect is comparable to that of the results obtained when the atomic oxygen density is multiplied by 2: the altitude of the DoLP max increases to $243 \mathrm{~km}$ with a maximum amplitude of $1.76 \%$. The width of the curve is larger though. However, the results of this simulation must be taken with caution because the electron densities and temperatures used here below $340 \mathrm{~km}$ are still those measured by the ESR during our campaign whilst in such stormy conditions, these parameters would be very different.

\subsubsection{Effect of the electron density and temperature profiles}

The electron population is divided in two parts: the thermal electrons and the suprathermal electrons. The thermal electrons are the ambient electron population, with energy of the order of $0.1 \mathrm{eV}$. The suprathermal electrons find their origin in the electron precipitation which change their pitch angle through elastic collisions and gradually lose their energy through ionisation, dissociation, excitation and heating whilst transported throughout the atmosphere. Eventually, the suprathermal electrons end up thermalised. In our case, a flux of precipitating electrons with a characteristic energy of $1 \mathrm{keV}$ loses its energy at all altitudes, producing a suprathermal electron flux at different energies and pitch angles. The heating mechanism is efficient at energies below $10 \mathrm{eV}$, i.e. at the same energies where the $\mathrm{O}^{1} \mathrm{D}$ state is produced. It is described through a Coulomb interaction. It can be parameterised and included in the transport equation as a continuous energy loss process which is described using a stopping or loss function $L(E)$ (in $\mathrm{eV} \mathrm{cm}^{2}$ ) (Swartz \& Nisbet 1972; Rees 1989):

$$
L(E, z)=\frac{5.39 \times 10^{-3}}{E^{0.94} n_{\mathrm{e}}^{0.03}(z)}\left(\frac{E-E_{\mathrm{th}}(z)}{E-0.53 E_{\mathrm{th}}(z)}\right)^{2.36},
$$

$E_{\mathrm{th}}(z)=8.61 \times 10^{-5} T_{\mathrm{e}}(z)$ is the thermal energy (in $\mathrm{eV}$ ), where $T_{\mathrm{e}}(z)$ is the electron temperature (in $\mathrm{K}$ ). Therefore, 


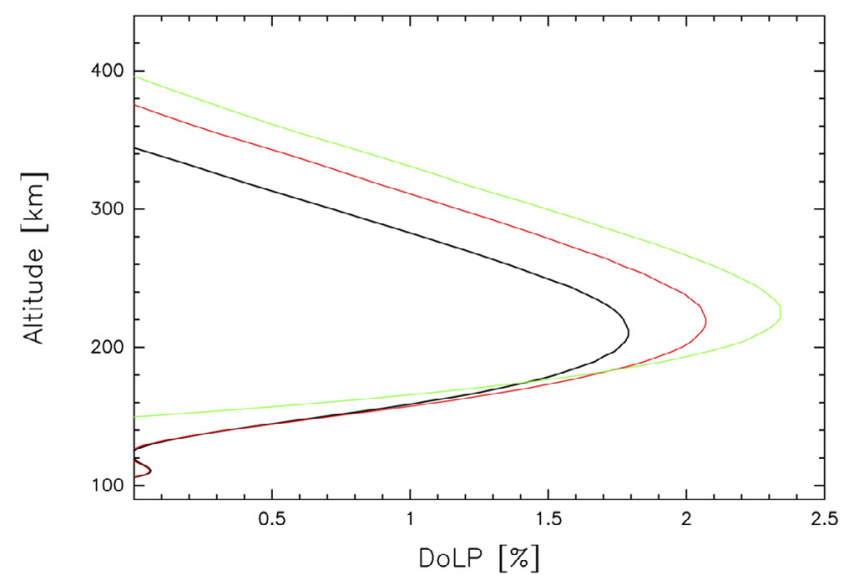

Fig. 11. Influence of the flux of precipitating electrons on the local DoLP characteristics. The black continuous line is the reference test case as in Figure 7. The red line corresponds to an isotropic flux with a characteristic energy of $1 \mathrm{keV}$ and a total energy flux of $0.02 \mathrm{erg} \mathrm{cm}^{-2} \mathrm{~s}^{-1}$. The green line is for a flux mainly forward with a characteristic energy of $500 \mathrm{eV}$ and a total energy flux $0.02 \mathrm{erg} \mathrm{cm}^{-2} \mathrm{~s}^{-1}$.

the electron density and temperature may influence the polarisation rate.

The results of changing the electron temperatures and densities are shown in Figure 10. Dividing the electron temperature by a factor of 2 has hardly any effect, and increasing it by a factor of 2 slightly enhances the value of the maximum local DoLP up to $1.9 \%$. The peak altitude remains the same, which is not surprising because the heating rate does not affect the neutral density. The influence of the variation of the electron density is shown as well. Similarly to the temperature, it is either multiplied or divided by a factor of 2 . The altitude of the peak DoLP and its amplitude vary slightly but the effect is much smaller than that due to the variation of the neutral atmosphere or the scattering phase function. Therefore, although the properties of the ionosphere influence the characteristics of the DoLP slightly they are not a major contributor. This is not surprising because an underlying assumption in Eq. (20) is that there is no dependence on the angle (the heating is isotropic) and so it cannot affect the anisotropy. However, it may be worth in the future to assess again this function in order to check this assumption.

\subsubsection{Effect of the precipitating flux of electrons}

In the previous sections, the electron flux at the top of the ionosphere was mainly field aligned (see Sect. 4.3). We conserve the same precipitation spectrum (centred around $1 \mathrm{keV}$ with an integral energy flux of $0.02 \mathrm{erg} \mathrm{cm}^{-2} \mathrm{~s}^{-1}$ ) but we use an isotropic angle distribution at the top of the atmosphere: the downward input flux is then spread over $2 \pi$. The results are shown in Figure 11. The effect is large, with a DoLP increasing up to $2.07 \%$ at $221 \mathrm{~km}$. This is due to the fact that most electrons cannot penetrate deep into the atmosphere because they enter it with a large pitch angle. Therefore, they are trapped at the altitude where the atomic oxygen is more abundant. The same behaviour occurs when we decrease the characteristic energy of the precipitating electrons from $1 \mathrm{keV}$ down to $500 \mathrm{eV}$. This value is considered as the mean energy of the polar rain, which is permanently precipitating in the polar cap. In this case, the DoLP increases to a maximum of
$2.34 \%$ at $225 \mathrm{~km}$. The reason is that electrons with a smaller energy do not penetrate as deep in the atmosphere. Therefore, the DoLP could also be useful to determine the pitch angle distribution of the precipitated electrons and their characteristic energy. The model was also run with a total energy flux of $1 \mathrm{erg} \mathrm{cm} \mathrm{cm}^{-2} \mathrm{~s}^{-1}$ instead of $0.02 \mathrm{erg} \mathrm{cm}^{-2} \mathrm{~s}^{-1}$ for the electrons. The result is not shown in Figure 11 because it cannot be distinguished from the test case. This may be understood when considering Eq. (1). The DoLP is a ratio which does not vary with the total energy. The total energy influences certainly the intensity of the red line but not the ratio. Therefore, in order to get a good idea of the precipitated flux, both measurements are needed, that of the intensity profile of the red line and that of its polarisation.

\subsubsection{Conclusion of this sensitivity study}

The results of the modelling are summarised in Table 1, with three parameters (maximum value of the DoLP, altitude of the maximum and height-integrated value of DoLP) listed for different conditions specified in the first column. The percentage difference from the test case is given for each simulation. The most significant parameters that influence the DoLP are the scattering phase function and the characteristics of the precipitating flux (characteristic energy, pitch angle distribution). The uncertainties in the neutral atmosphere mean that the effect of changes in the density of atomic oxygen can be disregarded.

The discovery of the red line polarisation raised some hope that it could become a new proxy for space weather studies, in particular for the monitoring of the upper atmosphere at high latitude. The study performed here shows that it is a very difficult task and would require very accurate polarisation measurements which are difficult to obtain with the weak signals at work. Another complication is that variation of several parameters can lead to the same value of the polarisation peak or altitude. It is now obvious that the polarisation of the red line should rather be considered as an additional parameter completing other measurements, such as the intensity of the emissions. However, interestingly, the polarisation of the oxygen atomic red line opens new prospects to re-examine the scattering function of the electrons, especially if the precipitated flux can be measured by other means. If the scattering phase function is precisely known, the polarisation could become a tool to constrain the estimation of the precipitating electron spectrum.

\section{Experimental determination of the linear polarisation}

In the modeling discussion (Sect. 3) we showed that the integrated DoLP ranges between $0.6 \%$ and $1.8 \%$ depending on various parameters. How does this compare to the measured polarisation? In February 2012, a coordinated campaign was organised in Svalbard using both SPP and the Eiscat Svalbard Radar (ESR). ESR was used to measure the ionospheric plasma parameters for estimation of the electron precipitation (flux). The modelled DoLP based on input from the ESR measurements was then compared to those measured with SPP.

\subsection{Observational conditions}

ESR is an Incoherent Scatter Radar located close to the city of Longyearbyen $\left(78.15^{\circ}\right.$ geographic latitude, $16.02^{\circ}$ geographic 
longitude). It is composed of two antennas: the first, with a diameter of $42 \mathrm{~m}$, is fixed and points along the local magnetic field line; the second, with a diameter of $32 \mathrm{~m}$, is fully steerable. Both antennas routinely measure the electron density, the electron and ion temperatures and the line-of-sight ion velocity profiles.

Barthélemy et al. (2011) showed that there is no polarisation when pointing the SPP along the magnetic field line. The initial idea was to point SPP and the $32 \mathrm{~m}$ ESR antenna in the same direction, then changing direction periodically in order to draw a map of the nightside polarisation angles. Unfortunately, the week prior to our campaign, a problem with the gearbox of the radar prevented us from moving the antenna more than once over the whole campaign. The antenna was in a safe mode, pointing in an unfortunate magnetic north direction above the city of Longyearbyen, where there might be some polarisation contamination due to city lights. During the whole campaign, only data from one night (from February 15 to 16) can fully serve our purpose: because of the good observational conditions, it was possible to move the antenna towards the magnetic west with an elevation of $45^{\circ}$ during a short period,

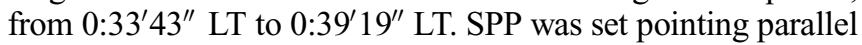
to this $32 \mathrm{~m}$ antenna. In this pointing direction, the lights from Longyearbyen are not scattered and cannot create artificial polarisation.

There was no cloud, no wind that blows crystal ice in the air, and a cold and constant temperature, between $-19^{\circ}$ and $-21^{\circ}$. The geomagnetic and solar conditions during that night were $\mathrm{Ap}=21, \mathrm{~F}_{10.7}=105$ with an 81-day average of 130 .

A set of auroras was observed before and after the period of the observations, but not in the direction of observation at the time of coordinated measurements between SPP and ESR.

\subsection{ESR measurements}

In Figure 12, the electron density, electron and ion temperatures and ion velocity are plotted as a function of altitude, as obtained from the ESR data (using the GUISDAP software, Lehtinen \& Huuskonen 1996). The full lines represent the average values during the period under study. The error bars represent the extrema measured during this period. We plot the data from both radars: the $42 \mathrm{~m}$ dish (parallel to the magnetic field) in blue and the $32 \mathrm{~m}$ dish (pointing west magnetic) in black. In the $\mathrm{E}$ region, the maximum electron density is about $9 \times 10^{9} \mathrm{~m}^{-3}$ at $150 \mathrm{~km}$ with the $42 \mathrm{~m}$ dish and $3 \times 10^{10} \mathrm{~m}^{-3}$ at $170 \mathrm{~km}$ with the $32 \mathrm{~m}$ dish. These values are very low, showing a very quiet atmosphere. The variability with height is high although the values themselves always remain in the range of a quiet ionosphere. This variability with altitude has two sources: first, the upper atmosphere varies but always with small electron density values. The relative variability with height is large because the values are small. Second, in the case of low electron density values, the noise in the radar measurement gets larger. It shows that both antennas measure quite comparable conditions. In the $\mathrm{F}$ region, the electron densities are almost the same in the two directions. This is especially true at the altitude of the maximum red line emission, i.e. around $220 \mathrm{~km}$.

The temperatures seem large, with electron values of about $1800 \mathrm{~K}$ in the E region in both antennas and about $1000 \mathrm{~K}$ for the ions. The low signal-to-noise ratio in the electron densities means that the fitting process used to estimate the temperatures produces large uncertainties.

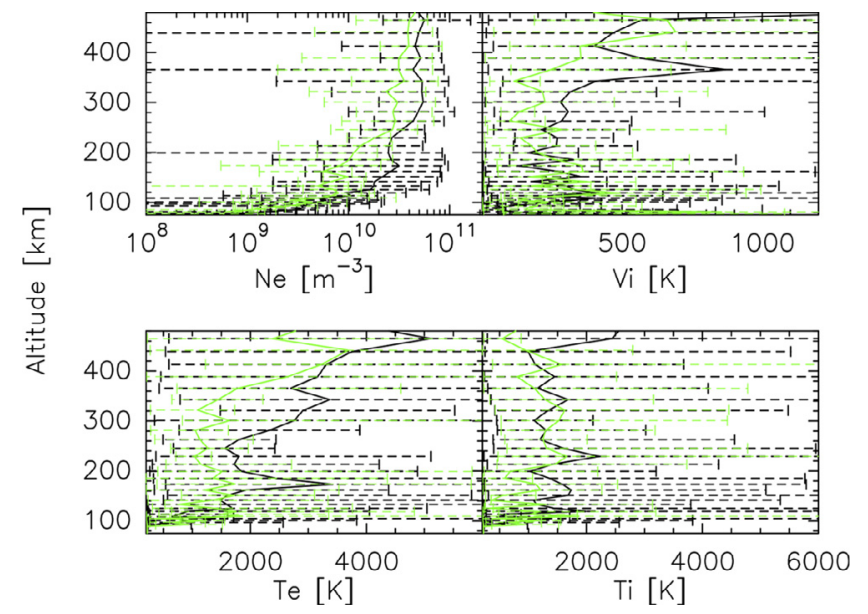

Fig. 12. ESR data averaged from $0: 33^{\prime} \mathrm{LT}$ to $0: 40^{\prime} \mathrm{LT}$. In green, the $42 \mathrm{~m}$ antenna (parallel to B). In black, the $32 \mathrm{~m}$ antenna (parallel to SPP field of view). The $32 \mathrm{~m}$ antenna is pointing magnetic west with an elevation of $45^{\circ}$. Upper panels: electron density (left) and line-of-sight ion velocity (right). Lower panels: electron temperature (left) and ion temperature (right).

Several features prevent us from using the upper atmosphere measurements (above about $340 \mathrm{~km}$ ). The electron density continuously increases in the $\mathrm{F}$ region with no prominent F2 peak, and the temperatures are very variable, with data exceeding $6000 \mathrm{~K}$, which in such low geomagnetic activity is an evidence of a quasi-empty ionosphere. The error bars are too large to give confidence in these upper atmosphere measurements. Therefore, above $340 \mathrm{~km}$, we ran IRI (Bilitza et al. 2012) to set artificially the electron temperature to $1150 \mathrm{~K}$, i.e. about $100 \mathrm{~K}$ above the exospheric temperature of $1060 \mathrm{~K}$ given by MSIS (Picone et al. 2002). These electron temperatures and densities are those used as first inputs in the modelling in Section 3.2. They constitute an anchor starting point, but the study of the parameters on the modelled polarisation lowers their importance.

\subsection{Deducing the stationary electron flux}

In order to determine the stationary electron flux, we compare the electron density profile obtained with the transport code to the electron density profile measured by the ESR. To obtain the former, first the electron production rate $P_{e}(z)\left(\mathrm{cm}^{-3} \mathrm{~s}^{-1}\right)$ is computed from the electron flux $\Phi(z, E, \theta)$. Then, assuming stationarity, the E-region electron density is computed from the electron production rate using the continuity equation given by

$$
N_{e}(z)=\sqrt{\frac{P_{e}(z)}{\alpha_{\mathrm{eff}}}}
$$

with an effective recombination coefficient (Banks \& Kockarts 1973; Roble et al. 1987) $\alpha_{\text {eff }}$ given by

$$
\alpha_{\text {eff }}=2.5 \times 10^{-6} \exp \left(-\frac{Z}{51.2}\right)
$$

where $z$ represents the altitude in kilometers.

The geometry of the measurements is shown in Figure 13. Using the electron density measured by the $42 \mathrm{~m}$ antenna 


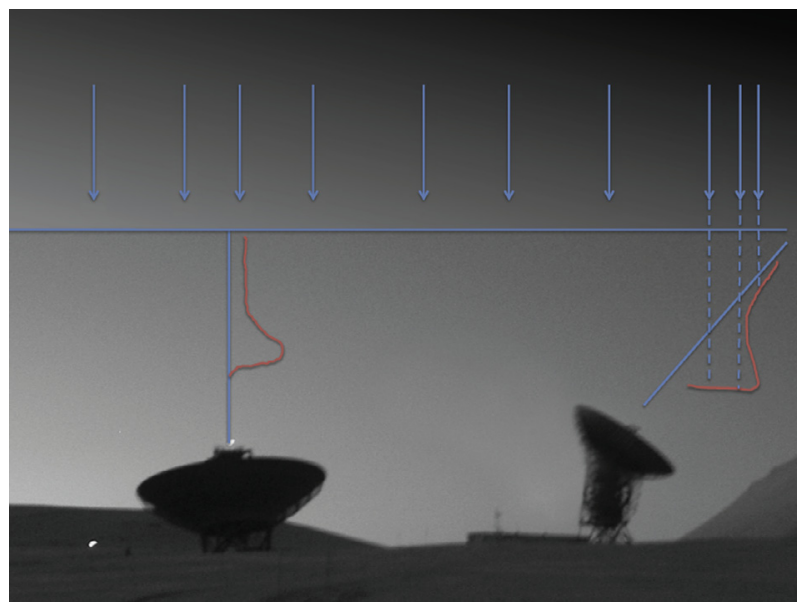

Fig. 13. Geometry of the observations. SPP points parallel to the $32 \mathrm{~m}$ dish (on the right of the picture). The arrows symbolise the precipitation. The blue lines show the line-of-sight. The red lines are the measured electron density profiles.

one can deduce the precipitation spectrum above the antenna. The situation is different with the $32 \mathrm{~m}$ antenna because the radar beam and the magnetic field line form an angle, i.e., the radar probes a series of spatially distributed and possibly different electron fluxes with different energy spectra. We therefore make a fit to the $42 \mathrm{~m}$ data to obtain an energy spectrum and energy flux. For the $32 \mathrm{~m}$ data we obtain an approximate value for the energy flux as a comparison. The ESR data show that in the entire volume in which measurements were taken the atmosphere is very quiet and may be considered uniform.

By fitting the measured and modelled electron density profiles, we obtain a precipitated flux centred around $1 \mathrm{keV}$ with an integral energy flux of $0.02 \mathrm{erg} \mathrm{cm}^{-2} \mathrm{~s}^{-1}$, i.e. $1.25 \times 10^{10} \mathrm{eV} \mathrm{cm}^{-2} \mathrm{~s}^{-1}$ for the $42 \mathrm{~m}$ dish and an integral energy flux 10 times higher $\left(0.2 \mathrm{erg} \mathrm{cm}^{-2} \mathrm{~s}^{-1}\right)$ to reproduce the profile measured with the $32 \mathrm{~m}$ antenna (again, this is not the electron precipitation above the pointing direction due to the antenna angle). These integral energy fluxes are very small compared to the normal energy fluxes in the auroral oval. Indeed, about $1 \mathrm{erg} \mathrm{cm}^{-2} \mathrm{~s}^{-1}$ is needed to produce visible aurora whilst a bright aurora requires more than $100 \mathrm{erg} \mathrm{cm}^{-2} \mathrm{~s}^{-1}$ (Vallance Jones 1992). However, such values are not unusual in the polar cap above Svalbard (Hardy et al. 1985). The measured and modelled densities are plotted in Figure 14.

The computed electron precipitated flux above the $42 \mathrm{~m}$ dish is given in Figure 2. The shape of the flux computed for the $32 \mathrm{~m}$ dish is the same as that of the $42 \mathrm{~m}$ antenna. Its energy flux is larger by a factor of about 10 . However, the fluxes deduced from these densities must not be taken as the real precipitated fluxes above the ionosphere, but only as fluxes that reproduce well the E-region electron density with this transport code, this neutral atmosphere and this set of cross-sections. In particular, the E-region and lower F-region altitudes were hit by precipitated electrons with energies larger than $300 \mathrm{eV}$ whilst precipitated electrons with lower energies are absorbed at higher altitudes. So, the low-energy tail of the electron spectra in Figure 2 is arbitrary.

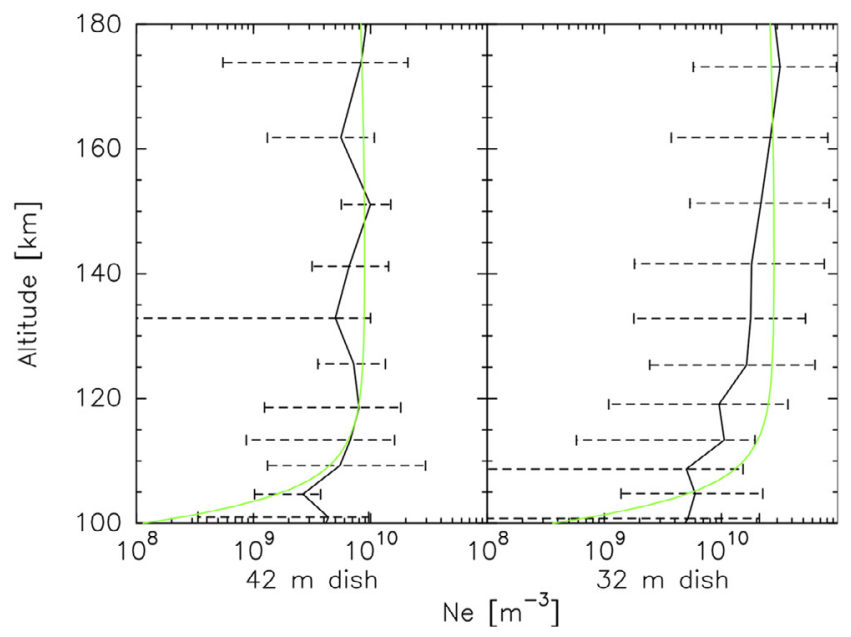

Fig. 14. The dark lines are the electron densities as in Figure 12 (upper left panel). The continuous green lines are the electron density deduced from the transport code. Left: the $42 \mathrm{~m}$ antenna (fixed, parallel to B). Right: the $32 \mathrm{~m}$ antenna (parallel to SPP field of view).

The precipitated electron flux deduced from the $42 \mathrm{~m}$ dish has been used as input in Section 3.2, and the $32 \mathrm{~m}$ was used in the sensitivity study in Section 3.3.

\subsection{SPP measurements}

SPP observed only the polarisation of the $630 \mathrm{~nm}$ atomic oxygen red line. The solar zenith angle was equal to $114^{\circ}$, i.e. larger than 108 degrees (which is the the limit for astronomical darkness) during the period of interest, so that no Rayleigh scattering could perturb the polarisation measurement. The red line intensity first increased (up to 250 counts per $50 \mathrm{~ms}$ ) then slowly decreased. After this period, the red line intensity remained smaller (less than 130 counts per $50 \mathrm{~ms}$ ). These were also very low values compared to the measurements made during preceding campaigns.

The data processing to compute the Degree of Linear Polarisation (DoLP) and the Angle of Linear Polarisation (AoLP) is detailed in the Appendix. The polarisation results are shown in Figure 15. The DoLP is quite stable during that period with an average of $1.92 \%$ and a standard deviation of $0.12 \%$ (or, considering the uncertainties, $1.9 \pm 0.1 \%$ ). The AoLP fluctuates around a mean value of $7.53^{\circ}$ with a standard deviation of $3.0^{\circ}$ but hardly shows a trend during this period. The value of the DoLP is relatively stable since, one hour earlier, it was measured at $2 \%$ with the same azimuth but at an elevation of $30^{\circ}$ (looking magnetic west, the angle between the line-of-sight and the magnetic field remains perpendicular).

These observations confirm that the polarisation remains measurable even in geomagnetically very quiet periods. In the previous studies, we observed that it decreases when the conditions become more active (Lilensten et al. 2013a).

\section{Comparison between modelled and experimental DoLP}

The DoLP measured by the SPP instrument has to be compared to the modelled line-of-sight integrated DoLP. 


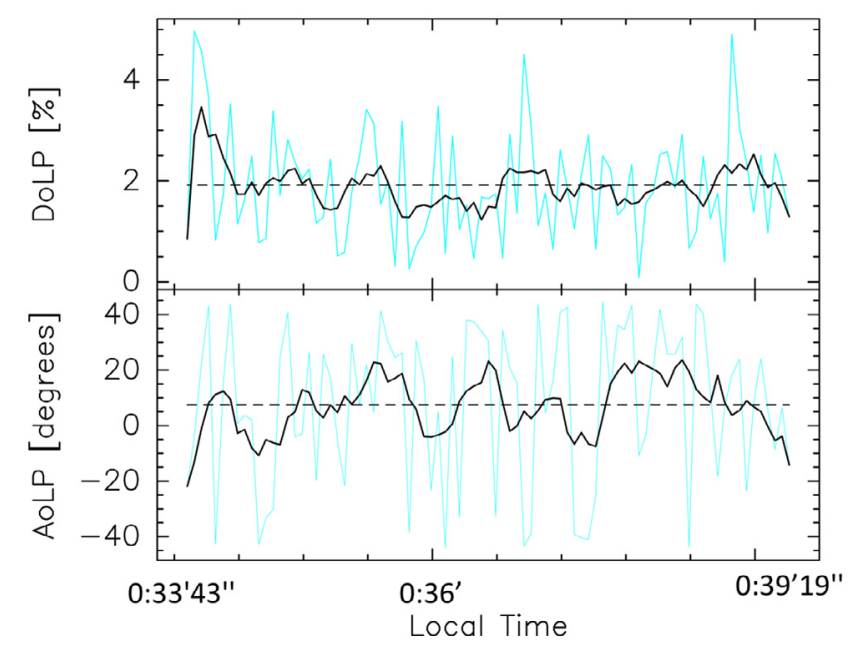

Fig. 15. Polarimetric observations of the auroral red line with SPP versus local time. The blue lines correspond to the instantaneous measurements whilst the black line is a moving average over $28 \mathrm{~s}$. The dashed lines represent the average over the whole period of observation. Upper panel: Degree of Linear Polarisation (DoLP). Lower panel: Angle of Linear Polarisation (AoLP).

The observed value is $1.9 \% \pm 0.1 \%$. For evaluating the theoretical line-of-sight integrated DoLP, a geometrical model is required, to determine the integration limits and the scattering angle. For the scattering angle $\Theta$, in the following we consider $\Theta=90^{\circ}$, because as described above the line-of-sight was West oriented, whereas the magnetic field line is North oriented. In the following, we investigate the possibility of experimental errors, and we discuss the final step of the theoretical DoLP computation, which is the determination of the integration limits.

\subsection{Errors sources}

The polarimeter data processing is given in the Appendix. It takes the instrumental polarisation into account. Its process - using the Mueller matrixes - is the standard for such instrumental data processing. The polarimeter has been calibrated before the experimental campaign in laboratory using a calibrated lamp at different polarisation angles to determine the instrumental polarisation. However, it is practically impossible to calibrate in the field, as it requires a calibration lamp whose use is impossible in the outdoor arctic conditions. Therefore, an error in the measurement cannot be excluded.

There are obvious errors in the ionospheric parameters and in the deduced precipitated electron flux. Figure 12 shows that the ESR data are very noisy due to the low values of the electron densities. The ESR data are not so important though in the above-modelled/measured study of polarisation because they are used only as starting test point. Indeed, by varying the parameters independently from the ESR measurements, we are not able in the current approach to have the modelled DoLP fit exactly the observed one.

Since the modelling only considers electron precipitation, this raises the question of other polarisation sources. Rayleigh scattering in the lower atmosphere issuing from parts of the auroral arc not intercepted by the SPP field of view has been studied in Barthélemy et al. (2011). In this study, we calculated the intensity and the polarisation due to the Rayleigh scattering using the all-sky images of the oxygen red line obtained from the $\mathrm{UiO}$ (University in Oslo) camera as well as the scattered city light or the light of the stars in the field of view. We used the formalism by Bucholtz (1959) and considered the anisotropy in the Rayleigh Scattering. We found that the Rayleigh scattering from other parts of the sky (including the city lights) cannot explain the observed DoLP nor contaminate it significantly.

Protons may be an additional source of polarised red line. A very detailed story of the discovery of the proton precipitations to the more recent observations and modelling can be found in Simon et al. (2007) and will not be repeated here. The emission of the atomic oxygen red line in proton aurora is addressed in Lummerzheim et al. (2001). This study shows that protons are efficient in creating the red line. In the present study, we have no space measurement of proton precipitation and the proton transport modelling is not included in the current set of codes. The presence of such proton precipitation could well explain the fact that the observed polarisation is higher than the modelled one, which only accounts for electron precipitation.

\subsection{The integration limits}

The DoLP measured by the SPP instrument has to be compared to the quotient of the integrations of $Q$ and $I$ along the line-of-sight, hence integrating their contributions at various altitudes (see Eq. (17)). Although $\langle$ DoLP $\rangle$ is a ratio of integrals, we called it the DoLP integrated over the line-of-sight in the preceding sections. But it is necessary to determine the limits $z_{\min }$ and $z_{\max }$, between which the integration has to be performed.

The two extreme cases are the curtain-like aurora (and corresponding curtain-like precipitation), on the one hand, and the completely diffuse aurora (with corresponding precipitation scattered in a large surface of the sky and reasonably spatially uniform), on the other hand. In the case of the curtain-like precipitation, $z_{\min }$ and $z_{\max }$ are very close. Then, the integrated DoLP is equal to the local DoLP, whose maximum value is $1.8 \%$. This value is in rather good agreement with the present observation. However, the fact that the two EISCAT antennas make similar measurements hardly supports this assumption.

It is clear from the modelling results that the integrated DoLP will be smaller than the maximum local DoLP of $1.8 \%$, and that the smallest possible theoretical DoLP will be obtained when $z_{\text {min }}=90 \mathrm{~km}$ and $z_{\max }=500 \mathrm{~km}$, which corresponds to a completely diffuse precipitation extending through the whole ionosphere. The DoLP integrated from $90 \mathrm{~km}$ to $500 \mathrm{~km}$ is $0.62 \%$, which is then the lowest limit for the theoretical DoLP. The present radar observation would favour this theoretical value.

As shown in previous observations (Lilensten et al. 2013a), the observed DoLP increases in the absence of aurora, which is consistent with our present case of an observed DoLP rather larger than the theoretical one.

Our results both theoretical and observed are encouraging towards an agreement, but further observations, in the presence of an aurora, have still to be performed.

\section{Conclusion}

In this paper, we provide a first theoretical modelling of the DoLP of the atomic oxygen red line produced by electron impacts due to electron precipitation only. We study the effects of different parameters. In the conditions of this theoretical 
study, we find that the modelled value integrated along the line-of-sight ranges between $0.6 \%$ and $1.8 \%$ depending on various parameters. The study of the relative effects of these parameters shows that:

- The DoLP maximum value and corresponding altitude are sensitive to the electron scattering phase function. In the past, the same scattering function has been used for all atmospheric species. This function (Porter et al. 1987) has been fitted on $\mathrm{N}_{2}$-electron collisions only, and for energies larger than $300 \mathrm{eV}$. This work shows that there is a need for a specific function for other atmospheric species, especially at low energy, between 2 and $10 \mathrm{eV}$, especially around the $\mathrm{O}^{1} \mathrm{D}$ excitation threshold. From the present study, it seems that at this energy, the distribution of the angles is intermediate between isotropic and forward peaked.

- The DoLP is sensitive to variations of the geomagnetic activity and to other thermospheric/ionospheric parameters: neutral atmosphere composition, precipitated electron flux. It could be used as a space weather tool to complement other measurements and proxies. The polarisation should not be understood as a geomagnetic proxy but as a tool to characterise the thermosphere which is still poorly constrained (Bruinsma et al. 2012). In order to use it for this purpose, the flux of precipitating particles must be estimated accurately by independent observations.

We then compare with measurements during a coordinated experiment in February 2012. We use the measurements from the incoherent scatter radar ESR and the polarimeter SPP. The measured DoLP is $1.9 \% \pm 0.1 \%$. We suggest different possibilities to explain the difference between modelling and measurements. However, only one very short set of data is available for this comparison, and none have been available since 2012. More observations are mandatory, using space observations to constrain the electron and possibly proton precipitation, and a set of ground instruments such as the ALIS network (Aso et al. 1998; Gustavsson 1998; Brändström 2003) and EISCAT (Safargaleev et al. 2009).

Acknowledgements. The present research project has been supported by the European Project COST MP1104 "Polarisation as a tool to study the Solar System and beyond" and the European FP7 Project ESPAS, Grant Agreement No: 283676 (NearEarth Space Data Infrastructure for e-Science). EISCAT is an international association supported by research organisations in China (CRIRP), Finland (SA), Japan (NIPR and STEL), Norway (NFR), Sweden (VR) and the United Kingdom (NERC). We thank D. Lorentzen and F. Sigernes at UNIS for their invaluable contributions. J. Moen was supported by the Research Council of Norway Grant 208006/F50. J. Lilensten and M. Barthélemy thank the institute IPEV for its continuous support. All the plots in this article have been made with the Gildas Greg software developed at the Institute of Metric Radio Astronomy (IRAM) and used in many international astronomical facilities. JL thanks his son for helping in the Figure 3. The editor thanks three anonymous referees for their assistance in evaluating this paper.

\section{References}

Aso, T., M. Ejiri, A. Urashima, H. Miyaoka, Å. Steen, U. Brändström, and B. Gustavsson. First results of auroral tomography from ALIS-Japan multi-station observations in March, 1995. Earth, Planets, and Space, 50, 81-86, 1998.

Banks, P.M., and G. Kockarts. Aeronomy, Springer, 1973.
Barthélemy, M., J. Lilensten, F. Pitout, C. Simon Wedlund, R. Thissen, et al. Polarisation in the auroral red line during coordinated EISCAT Svalbard Radar/optical experiments. Ann. Geophys., 29, 1101-1112, 2011.

Bilitza, D., S.A. Brown, M.Y. Wang, J.R. Souza, and P.A. Roddy. Measurements and IRI model predictions during the recent solar minimum. J. Atmos. Sol. Terr. Phys., 86, 99-106, 2012.

Bommier, V., S. Sahal-Brechot, J. Dubau, and M. Cornille. The theoretical impact polarization of the O I 6300 A red line of Earth Aurorae. Ann. Geophys., 29, 71-79, 2011.

Brändström, B.U.E PhD thesis, The auroral large imaging system: design, operation and scientific results, Institutet fr rymdfysik (IRF) Sci. Rep., 279, IRF, Kiruna, Sweden, 2003.

Brink, D.M., and G.R. Satchler. Angular Momentum, 3rd edn., Clarendon Press, Oxford, 1994.

Bruinsma, S.L., N. Sanchez-Ortiz, E. Olmedo, and N. Guijarro. Evaluation of the DTM-2009 thermosphere model for benchmarking purposes. J. Space Weather Space Clim., 2, A04, 2012, DOI: $10.1051 / \mathrm{swsc} / 2012005$.

Bucholtz, A. Rayleigh-scattering calculations for the terrestrial atmosphere. Appl. Opt., 34, 2765, 1959.

Charvin, P. Étude de la polarisation des raies interdites de la couronne solaire. Application au cas de la raie verte $\lambda 5303$. Annales d'Astrophysique, 28, 877, 1965.

Clarke, D. Stellar Polarimetry, Wiley, ISBN: 978-3-527-40895-5, 2010.

Culot, F., C. Lathuillère, and J. Lilensten. Influence of geomagnetic activity on the O I 630.0 and $557.7 \mathrm{~nm}$ dayglow. J. Geophys. Res. [Space Phys.], 110, 1304-13013, 2005.

Goldstein, D. Polarized light, Marcel Dekker, New York, NY, 2003.

Gustavsson, B. Tomographic inversion for ALIS noise and resolution. J. Geophys. Res., 103, 26621-26632, 1998.

Hardy, D.A., M.S. Gussenhoven, and E. Holeman. A statistical model of auroral electron precipitation. J. Geophys. Res., 90, 4229-4248, 1985.

Landi Degl'Innocenti, E. Polarization in spectral lines. III Resonance polarization in the non-magnetic, collisionless regime. Sol. Phys., 91, 1-26, 1984.

Landi Degl'Innocenti, E., and M. Landolfi. Polarization in spectral lines, vol. 307 of Astrophysics and Space Science Library, Kluwer Academic Publishers, 2004

Lehtinen, M.S., and A. Huuskonen. General incoherent scatter analysis and GUISDAP. J. Atmos. Terr. Phys., 58, 435-452, 1996.

Lilensten, J., and L.R. Cander. Calibration of the TEC derived from GPS measurements and from ionospheric models using the EISCAT radar. J. Atmos. Sol. Terr. Phys., 65, 833-842, 2003.

Lilensten, J., J. Moen, M. Barthélemy, R. Thissen, C. Simon, D.A. Lorentzen, O. Dutuit, P.O. Amblard, and F. Sigernes. Polarization in aurorae: a new dimension for space environments studies. Geophys. Res. Lett., 35, 8804, 2008.

Lilensten, J., M. Barthélémy, P.O. Amblard, H. Lamy, C.S. Wedlund, et al. The thermospheric auroral red line polarization: confirmation of detection and first quantitative analysis. J. Space Weather Space Clim., 3, A01, 2013a, DOI: 10.1051/swsc/2012023.

Lilensten, J., C. Simon Wedlund, M. Barthélémy, R. Thissen, D. Ehrenreich, G. Gronoff, and O. Witasse. Dications and thermal ions in planetary atmospheric escape. Icarus, 222, 169-187, $2013 \mathrm{~b}$.

Lummerzheim, D., and J. Lilensten. Electron transport and energy degradation in the ionosphere: evaluation of the numerical solution, comparison with laboratory experiments and auroral observations. Ann. Geophys., 12, 1039-1051, 1994.

Lummerzheim, D., M.H. Rees, and H.R. Anderson. Angular dependent transport of auroral electrons in the upper atmosphere. Planet. Space Sci., 37, 109-129, 1989.

Lummerzheim, D., M. Galand, J. Semeter, M.J. Mendillo, M.H. Rees, and F.J. Rich. Emission of O I $(630 \mathrm{~nm})$ in proton aurora. J. Geophys. Res., 106, 141-148, 2001.

Martinez Herrero, R., P.M. Mejias, and G. Piquero. Characterization of partially polarized light field. Springer Series on Optical Sciences, 147, 2009, DOI: 10.1007/978-3-642-01327-0. 
Moore, C.E., and J.W. Gallagher. Tables of spectra of hydrogen, carbon, nitrogen, and oxygen atoms and ions, CRC Press, Boca Raton, FL, 1993.

Opal, C., W. Peterson, and E. Beaty. Measurements of secondaryelectron spectra produced by electron impact ionization of a number of simple gases. J. Chem. Phys., 55, 4100, 1971.

Picone, J.M., A.E. Hedin, D.P. Drob, and A.C. Aikin. NRLMSISE00 empirical model of the atmosphere: statistical comparisons and scientific issues. J. Geophys. Res. [Space Phys.], 107, 1468, 2002.

Porter, H.S., F. Varosi, and H.G. Mayr. Iterative solution of the multistream electron transport equation. I - Comparison with laboratory beam injection experiments. J. Geophys. Res., 92, 5933-5959, 1987.

Press, W.H., S.A. Teukolsky, W.T. Vetterling, and B.P. Flannery. Numerical recipes in FORTRAN. The art of scientific computing, 2nd edn., University Press, Cambridge, 1992.

Rees, M.H. Physics and chemistry of the upper atmosphere, University Press, Cambridge, ISBN: 0521368480, 1989.

Roble, R.G., E.C. Ridley, and R.E. Dickinson. On the global mean structure of the thermosphere. J. Geophys. Res., 92, 8745-8758, 1987.

Safargaleev, V.V., T.I. Sergienko, A.E. Kozlovsky, I. Sandahl, U. Brändström, and D.N. Shibaeva. Electric field enhancement in an auroral arc according to the simultaneous radar (EISCAT) and optical (ALIS) observations. Geomag. Aeron., 49, 353-367, 2009.
Sahal-Bréchot, S. Role of collisions in the polarization degree of the forbidden emission lines of the Solar Corona. II - Depolarization by electron impact and calculation of the polarization degree of the Green line of Fe XIV. Astron. Astrophys., 36, 355-363, 1974.

Simon, C., J. Lilensten, J. Moen, J. Holmes, Y. Ogawa, K. Oksavik, and W. Denig. TRANS4: a new coupled electron/proton transport code comparison to observations above Svalbard using ESR, DMSP and optical measurements. Ann. Geophys., 25, 661-673, 2007.

Solomon, S.C., and V.J. Abreu. The $630 \mathrm{~nm}$ dayglow. J. Geophys. Res., 94, 6817-6824, 1989.

Stenflo, J. Solar Magnetic Fields: Polarized Radiation Diagnostics, vol. 189, Kluwer Academic Pub.; Astrophysics and Space Science Library (ASSL), 1994.

Swartz, W.E., and J.S. Nisbet. Revised calculations of the f region ambient electron heating by photoelectrons. J. Geophys. Res., 77, 6259-6277, 1972.

Vallance Jones, A. Historical review of great auroras. Can. J. Phys., 70, 479-487, 1992.

Wiscombe, W., and G. Gums. The backscattered fraction in twostream approximations. Genesis, 2, 11, 1998.

Witasse, O., J. Lilensten, C. Lathuillere, and B. Pibaret. Meridional thermospheric neutral wind at high latitude over a full solar cycle. Ann. Geophys., 16, 1400-1409, 1998.

Witasse, O., J. Lilensten, C. Lathuillère, and P.-L. Blelly. Modeling the OI 630.0 and $557.7 \mathrm{~nm}$ thermospheric dayglow during EISCAT-WINDII coordinated measurements. J. Geophys. Res., 104, 24639-24656, 1999.

Cite this article as: Lilensten J, Bommier V, Barthélemy M, Lamy H, Bernard D, et al. The auroral red line polarisation: modelling and measurements. J. Space Weather Space Clim., 5, A26, 2015, DOI: 10.1051/swsc/2015027. 


\section{Appendix: Correction of the instrumental polarisation}

SPP introduces instrumental polarisation and modifies the state of the polarisation of the incident light. In Lilensten et al. (2013a), we removed the effect of the instrumental polarisation using the Mueller formalism (Stenflo 1994; Goldstein 2003; Martinez Herrero et al. 2009). This data processing has been improved, so that we provide here the new development. We determined a Mueller matrix $\mathbf{M}_{4}$ (e.g. Clarke 2010) proportional to:

$$
\mathbf{M}_{4}=\left(\begin{array}{cccc}
1 & P_{i} & 0 & 0 \\
P_{i} & 1 & 0 & 0 \\
0 & 0 & R & 0 \\
0 & 0 & 0 & R
\end{array}\right)
$$

where $P$ is the DoLP, and $R=\sqrt{1-P_{i}^{2}}$. In the following, the index o stands for "observed", $\mathrm{r}$ for "real" and i for "instrumental", $\theta$ the AoLP. The initial state of polarisation, hereafter referred to as "real", is described by the Stokes vector $\mathbf{S}_{\mathbf{r}}$

$$
\mathbf{S}_{\mathbf{r}}=\left(\begin{array}{c}
I_{r} \\
Q_{r} \\
U_{r} \\
V_{r}
\end{array}\right)
$$

whilst the observed polarisation is described by the Stokes vector $\mathbf{S}_{\mathbf{o}}$

$$
\mathbf{S}_{\mathbf{0}}=\left(\begin{array}{c}
1 \\
P_{\mathrm{o}} \\
0 \\
0
\end{array}\right)
$$

Circular polarisation is null because a linear polariser is used $\left(\mathrm{V}_{\mathrm{o}}=0\right)$. In Lilensten et al. (2013a), the observed direction of polarisation $\theta_{\mathrm{o}}$ is chosen as reference axis for the linear polarisation and the relation between $\mathbf{S}_{\mathbf{o}}$ and $\mathbf{S}_{\mathbf{r}}$ is given by

$$
\mathbf{S}_{\mathbf{0}}=\mathbf{R}_{4}^{-1} \mathbf{M}_{4} \mathbf{R}_{4} \mathbf{S}_{\mathbf{r}}
$$

where $R_{4}$ is the rotation matrix

$$
\mathbf{R}_{4}=\left(\begin{array}{cccc}
1 & 0 & 0 & 0 \\
0 & \cos 2 \alpha & \sin 2 \alpha & 0 \\
0 & -\sin 2 \alpha & \cos 2 \alpha & 0 \\
0 & 0 & 0 & 1
\end{array}\right)
$$

In the following, $S a=\sin 2 \alpha, C a=\cos 2 \alpha$, and $R=\sqrt{1-P_{i}^{2}}$. The real Stokes vector $\mathbf{S}_{\mathbf{r}}$ was then obtained by inverting Eq. (A1):

$$
S_{r}=R_{4}^{-1} M_{4}^{-1} R_{4} S_{0}=\Gamma_{4} S_{0}
$$

Allowing to compute a DoLP and an AoLP. The DoLP writes $P_{r}=\frac{N}{D}$ with

$$
N=\sqrt{P_{o}^{2}+P_{i}^{2}-2 P_{o} P_{i} C a-P_{o}^{2} P_{i}^{2} S a^{2}}
$$

and

$$
D=1-P_{o} P_{i} C a
$$

The real AoLP is deduced from the two following equations

$$
\begin{aligned}
& \cos \left(2 \theta_{r}\right)=\frac{Q_{r} / I_{r}}{P_{r}}=\frac{-\mathrm{CaPi}_{+} P_{o} \mathrm{Ca}^{2}+P_{o} S a^{2} \sqrt{1-P_{i}^{2}}}{N} \\
& \sin \left(2 \theta_{r}\right)=\frac{U_{r} / I_{r}}{P_{r}}=\frac{-\operatorname{SaP}_{i}+P_{o} \operatorname{CaSa}\left(1-\sqrt{1-P_{i}^{2}}\right)}{N}
\end{aligned}
$$

and applying the adequate quadrant correction according to the signs of $\cos \left(2 \theta_{r}\right)$ and $\sin \left(2 \theta_{r}\right)$. 
However, this development suffers an inadequacy. Indeed, if the instrumental DoLP $P_{i}=0$, there is no reference frame. The computed "real" AoLP becomes $90^{\circ}$ whatever the observed AoLP. The reason is that this formalism applies to a fully polarised signal, i.e. upstream the polarisation lens. In order to account for this rotating lens, we must re-write Eq. (A1) as:

$$
\mathbf{S}_{\mathbf{0}}=\Delta_{\mathbf{4}} \mathbf{R}_{4}^{-1} \mathbf{M}_{\mathbf{4}} \mathbf{R}_{\mathbf{4}} \mathbf{S}_{\mathbf{r}}
$$

where $\boldsymbol{\Delta}_{\mathbf{4}}$ represents the rotation over time of the polarisation lens:

$$
\mathbf{\Delta}_{\mathbf{4}}=\left(\begin{array}{cccc}
1 & \cos \omega t & \sin \omega t & 0 \\
\cos \omega t & (\cos \omega t)^{2} & \cos \omega t \sin \omega t & 0 \\
\sin \omega t & \cos \omega t \sin \omega t & (\sin \omega t)^{2} & 0 \\
0 & 0 & 0 & 0
\end{array}\right)
$$

$\omega$ is the pulsation, equal to $\frac{\pi}{2} \mathrm{~s}^{-1}$ since a full turn is performed in $4 \mathrm{~s}$. Developing Eq. (A5) over the first line, we obtain:

$$
I_{\mathrm{o}}(t)=A(t) I_{r}+B(t) Q_{r}+C(t) U_{r},
$$

where

$$
\left\{\begin{array}{l}
A(t)=1+\cos \omega t c a P i+\sin \omega t s a P i \\
B(t)=C a P i+\cos \omega t\left(C a^{2}+R S a^{2}\right)+\sin \omega t C a S a(1-R) \\
C(t)=S a P i+\cos \omega t C a S a(1-R)+\sin \omega t\left(S a^{2}+R C a^{2}\right)
\end{array} .\right.
$$

There are 80 observed $I_{\mathrm{o}}(t)$ every $4 \mathrm{~s}$. A Levenberg-Marquardt method is used (Press et al. 1992) to deduce the Stokes parameters $I_{r}$, $Q_{r}$ and $U_{r}$ through a mean square fitting. With this formalism, the instrumental polarisation may be null. In that case, $I_{\mathrm{o}}$ simply becomes:

$$
I_{o}(t)=I_{r}+\cos \omega t Q_{r}+\sin \omega t U_{r}
$$

We checked that this new signal processing does not change the conclusions in Lilensten et al. (2013a). 